\title{
MFN2 ameliorates cell apoptosis in a cellular model of Parkinson's disease induced by rotenone
}

 \\ ${ }^{1}$ Department of Neurology, Nanjing First Hospital, Nanjing Medical University, Nanjing, Jiangsu 210006; \\ ${ }^{2}$ Department of Neurology, Jiangyin People's Hospital, Nanjing Medical University, Jiangyin, Jiangsu 214400; \\ ${ }^{3}$ Department of Neurology, Huai'an First People's Hospital, Nanjing Medical University, Huai'an, Jiangsu 223300, P.R. China
}

Received March 26, 2018; Accepted July 13, 2018

DOI: $10.3892 /$ etm.2018.6595

\begin{abstract}
A number of studies indicated that apoptosis, a specific type of programmed cell death, contributed to the loss of dopaminergic neurons during progression of Parkinson's disease (PD). Previously, the authors of the present study demonstrated that apoptosis of dopaminergic neurons was mainly achieved via the mitochondria-mediated apoptosis pathway, however, the precise molecular mechanisms remain to be elucidated. The present study aimed to determine whether mitofusin-2 (MFN2), a mitochondrial protein, participated in the apoptosis of dopaminergic neurons in a cellular model of PD induced by rotenone. The present study demonstrated that the expression of MFN2 was relatively stable following treatment with rotenone. Lentiviral knockdown and overexpression experiments for the first time, to the best of the authors knowledge, revealed that MFN2 prevented rotenone-induced cell death by amelioration of apoptosis. These results revealed a protective role of MFN2 against apoptosis in an in vitro model of PD and may be used to establish MFN2 as a potential therapeutic target for the treatment of this disease.
\end{abstract}

\section{Introduction}

As the second most common type of neurodegenerative disorder, Parkinson's disease (PD) is characterized by evident motor symptoms including rigidity, bradykinesia, resting tremor and postural instability (1). These symptoms are mainly due to the selective dopaminergic neuron loss within basal ganglia structures (2).

Correspondence to: Professor Ying-Dong Zhang or Dr Teng Jiang, Department of Neurology, Nanjing First Hospital, Nanjing Medical University, 68 Changle Road, Nanjing, Jiangsu 210006, P.R. China E-mail: zhangyingdong@aliyun.com

E-mail: jt870918@163.com

*Contributed equally

Key words: mitofusin-2, apoptosis, rotenone, SH-SY5Y cells, Parkinson's disease
Previous studies indicated that apoptosis, a specific type of programmed cell death, contributed to the loss of dopaminergic neurons during PD progression $(3,4)$. In mammalian cells, apoptosis is closely modulated by two different signaling pathways including the receptor-dependent apoptotic pathway and the mitochondria-mediated apoptotic pathway (5). Previously, the authors of the present study demonstrated that apoptosis of dopaminergic neurons was primarily achieved via the mitochondria-mediated apoptotic pathway (6), however, the precise molecular mechanisms remain to be elucidated.

A member of the family of GTPases, mitofusin-2 (MFN2), is widely distributed in numerous tissues including heart, kidney, liver and brain (7). At the subcellular level, MFN2 is located predominantly in the outer membrane of the mitochondria, and is required for mitochondrial fusion, integrity and metabolism $(8,9)$. Previous reports suggested that MFN2 was involved in the process of mitochondria-mediated apoptosis and, therefore, contributed to the pathogenesis of several neurological disorders including Charcot-Marie-Tooth disease (CMT), ischemic stroke and Alzheimer's disease (10-12). However, the role of MFN2 in the apoptosis of dopaminergic neurons during the progression of PD remains unclear.

The present study therefore aimed to assess whether MFN2 participates in dopaminergic neuronal apoptosis using a cellular model of PD induced by rotenone (13).

\section{Materials and methods}

Cell culture. A human neuroblastoma cell line SH-SY5Y was purchased from American Type Culture Collection (Manassas, VA, USA; stock no. CRL-2266). Cells were cultured in RPMI 1640 medium (Thermo Fisher Scientific, Inc., Waltham, MA, USA) supplemented with $10 \%$ fetal calf serum (Thermo Fisher Scientific, Inc.) containing $2 \mathrm{mM}$ glutamine, $100 \mathrm{U} / \mathrm{ml}$ penicillin and $100 \mu \mathrm{g} / \mathrm{ml}$ streptomycin in a humidified atmosphere of $5 \% \mathrm{CO}_{2}$ at $37^{\circ} \mathrm{C}$, as previously described (13).

Lentiviral particles and cell transduction. For MFN2 knockdown and overexpression, lentiviral particles containing MFN2 short hairpin (sh) RNA (cat. no. sc-43928-V), lentiviral particles encoding human wild-type MFN2 cDNA (sc-400536-LAC) and their control lentiviral particles (cat. nos. sc-108080 and sc-437282, respectively) were purchased from Santa Cruz 
Biotechnology, Inc. (Dallas, TX, USA). The cell transduction was performed as previously described (14). The efficiency of transduction was determined by western blotting $72 \mathrm{~h}$ later as described below.

Rotenone treatment. SH-SY5Y cells were treated with vehicle (0.01\% DMSO) and rotenone (Sigma-Aldrich; Merck KGaA, Darmstadt, Germany; 20, 100 or $500 \mathrm{nM}$ dissolved in $0.01 \%$ DMSO) for $12 \mathrm{~h}$, as previously described (15). Subsequently, the cells were collected and used for the experiments described below.

Cell viability assay. The viability of SH-SY5Y cells was assessed by the MTT method, as previously described (4). The SH-SY5Y cells were seeded at a density of $1.5 \times 10^{5}$ cells $/ \mathrm{cm}^{2}$ and received the indicated treatment. Subsequently, MTT was added to the culture medium to reach a final concentration of $0.5 \mathrm{mg} / \mathrm{ml}$. After incubation at $37^{\circ}$ for $4 \mathrm{~h}$, the culture medium containing MTT was removed. DMSO was added into each well and the absorbance was measured at a wavelength of $490 \mathrm{~nm}$ using a microplate reader.

Colorimetric assay for caspase-3 activity. A colorimetric assay was carried out as previously described (3). The SH-SY5Y cells were seeded at a density of $1.5 \times 10^{5}$ cells $/ \mathrm{cm}^{2}$. After receiving the indicated treatment, the cells were harvested and lysed in an extraction buffer (included in the caspase-3 colorimetric assay kit mentioned below). The activity of caspase-3 was detected by a colorimetric assay kit (cat. no. ab39401; Abcam, Cambridge, UK) according to the manufacturer's protocol.

Western blotting. Western blotting was carried out as previously described (16). The SH-SY5Y cells were seeded at a density of $1.5 \times 10^{5}$ cells $/ \mathrm{cm}^{2}$ and received the indicated treatment. Subsequently, the cells were harvested and lysed in an extraction buffer containing complete protease inhibitor cocktail. Samples with equal amounts of protein were separated via SDS-PAGE on a $10 \%$ gel, transferred to nitrocellulose membranes and blocked with $5 \%$ bovine serum albumin (Thermo Fisher Scientific, Inc.). Following washing, membranes were incubated with a primary antibody against MFN2 (cat. no. ab101055; 1:500; Abcam) at $4^{\circ} \mathrm{C}$ overnight, washed again and incubated with a horseradish peroxidase (HRP)-conjugated secondary antibody (cat. no. A0208; 1:1,500; Beyotime Institute of Biotechnology, Haimen, China) for $2 \mathrm{~h}$. Following washing, protein bands were detected with a chemiluminescent HRP substrate and exposed to an $\mathrm{X}$-ray film. The signal intensity of primary antibody binding was analyzed using Quantity One software 4.6.2 (Bio-Rad Laboratories, Inc., Hercules, CA, USA) and normalized to a loading control GAPDH (cat. no. sc-47724; 1:1,000; Santa Cruz Biotechnology, Inc.).

Reverse transcription-quantitative polymerase chain reaction $(R T-q P C R)$. The SH-SY5Y cells were seeded at a density of $1.5 \times 10^{5}$ cells $/ \mathrm{cm}^{2}$ and received the indicated treatment. Subsequently, total RNA was extracted from SH-SY5Y cells using TRIzol reagent (Invitrogen; Thermo Fisher Scientific, Inc.). Equal amounts of total RNA were reverse transcribed in a final volume of $10 \mu \mathrm{l}$ with random primers under standard conditions (described in the manufacturer's protocol) using the PrimeScript ${ }^{\circledR}$ RT Master Mix (Takara Biotechnology Co., Ltd., Dalian, China). Reverse transcription reaction was carried out as previously described (17). Subsequently, qPCR reactions were performed with SYBR ${ }^{\circledR}$ Premix Ex Taq ${ }^{\mathrm{TM}}$ (Takara Biotechnology Co., Ltd.) and a specific primer (forward: 5'-TGGCTCAAGACTATAAGCTGCG-3', reverse: 5'-GAG GACTACTGGAGAAGGGTGG-3') to detect MFN2 mRNA expression levels. The thermo cycling conditions were the same as previously described (17). GAPDH (forward: 5'-AAG GTGAAGGTCGGAGTCAAC-3', reverse: 5'-GGGGTCATT GATGGCAACAATA-3') was used as an internal control. Relative levels were determined using the $2^{-\Delta \Delta \mathrm{Cq}}$ method (18).

Statistical analysis. Data are presented as the mean \pm standard deviation of at least four independent experiments. Statistical significance was detected by one-way analysis of variance followed by Turkey's post hoc test using SPSS software 18.0 (SPSS, Inc., Chicago, IL, USA). $\mathrm{P}<0.05$ was considered to indicate a statistically significant difference.

\section{Results}

Apoptosis contributes to the decrease in viability of $\mathrm{SH}-\mathrm{SY} 5 \mathrm{Y}$ cells induced by rotenone. The effects of rotenone on the viability of SH-SY5Y cells were assessed in the present study. Cells were incubated with different doses of rotenone $(20$, 100 and $500 \mathrm{nM}$ ), and MTT assay was used to evaluate cell viability after $12 \mathrm{~h}$. Rotenone (100 and $500 \mathrm{nM})$ significantly reduced the viability of $\mathrm{SH}-\mathrm{SY} 5 \mathrm{Y}$ cells by $38.3 \%(\mathrm{P}<0.05)$ and $45.6 \%(\mathrm{P}<0.05)$, respectively (Fig. 1A). This result implied that rotenone induced cell death in a dose-dependent manner. To investigate whether apoptosis contributed to the rotenone-induced cell death, activity of caspase-3, a crucial executioner of apoptosis, was detected using a colorimetric assay kit. Rotenone (100 and $500 \mathrm{nM})$ significantly increased caspase-3 activity 2.91-fold $(\mathrm{P}<0.05)$ and 3.47 -fold $(\mathrm{P}<0.05)$, respectively (Fig. 1B). These observations indicated that apoptosis contributed to the death of SH-SY5Y cells induced by rotenone.

Expression of MFN2 in SH-SY5Y cells is stable following treatment with rotenone. The effect of rotenone on the expression of MFN2 was detected in SH-SY5Y cells. Treatment with rotenone $(20,100$ and $500 \mathrm{nM})$ did not significantly affect the mRNA and protein expression levels of MFN2 compared with the vehicle control (Fig. 2A-C).

Knockdown of MFN2 exacerbates apoptosis induced by rotenone in $\mathrm{SH}-\mathrm{SY} 5 \mathrm{Y}$ cells. To further investigate the role of MFN2 in rotenone-induced apoptosis, a lentiviral transduction strategy was used to knock down MFN2 expression in $\mathrm{SH}-\mathrm{SY} 5 \mathrm{Y}$ cells. The alterations in MFN2 protein expression levels were confirmed by western blotting (Fig. 3A and B). Subsequently, cells were incubated with $100 \mathrm{nM}$ rotenone for $12 \mathrm{~h}$. Knockdown of MFN2 further increased the activity of caspase-3 1.72-fold $(\mathrm{P}<0.05)$ in $\mathrm{SH}-\mathrm{SY} 5 \mathrm{Y}$ cells following treatment with rotenone, compared with the control shRNA group (Fig. 3C). Furthermore, MFN2 knockdown further reduced the viability of $\mathrm{SH}-\mathrm{SY} 5 \mathrm{Y}$ cells by $52 \%(\mathrm{P}<0.05)$ following 

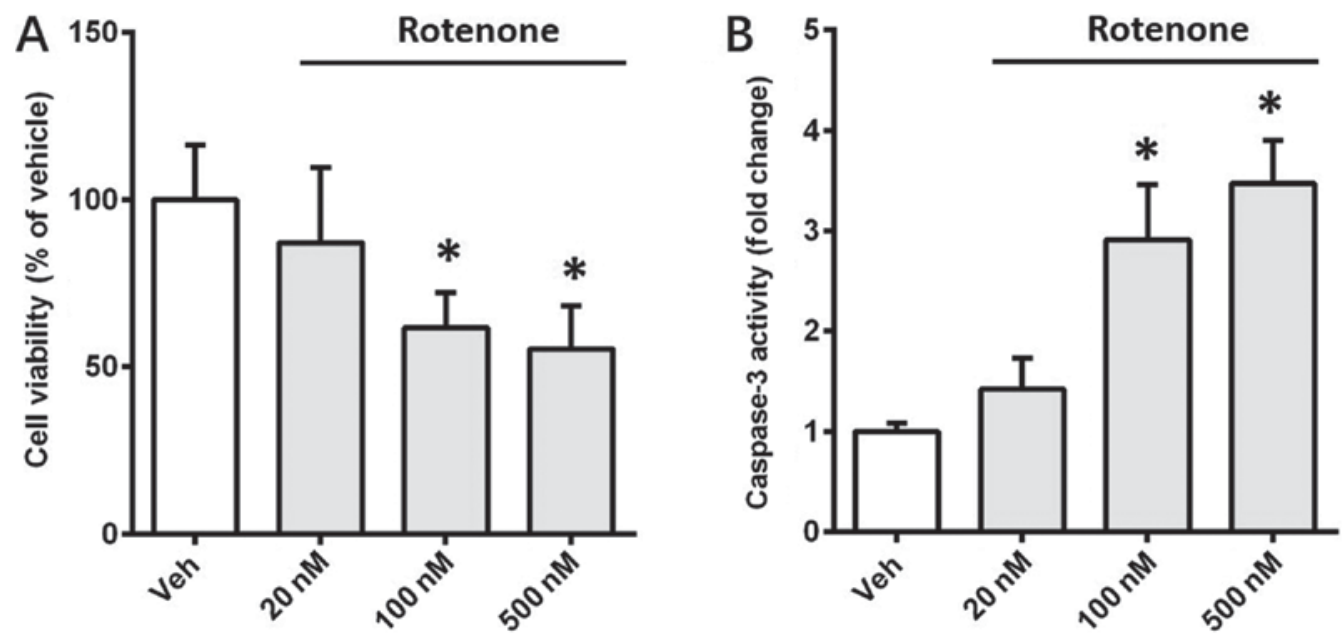

Figure 1. Apoptosis contributes to the death of SH-SY5Y cells induced by rotenone. SH-SY5Y cells were incubated with different doses of rotenone (20, 100 and $500 \mathrm{nM}$ ) for $12 \mathrm{~h}$. (A) MTT assay was used to evaluate the viability of SH-SY5Y cells. Data are presented as a percentage of the vehicle control group. (B) A colorimetric assay was used to detect the activity of caspase-3 in SH-SY5Y cells. Data are presented as a fold change relative to the vehicle control group. All figures are representative of at least four independent experiments. All data were analyzed by one-way analysis of variance followed by Tukey's post hoc test. Values are presented as the mean \pm standard deviation. " $\mathrm{P}<0.05$ vs. the vehicle control group. Veh, vehicle control.
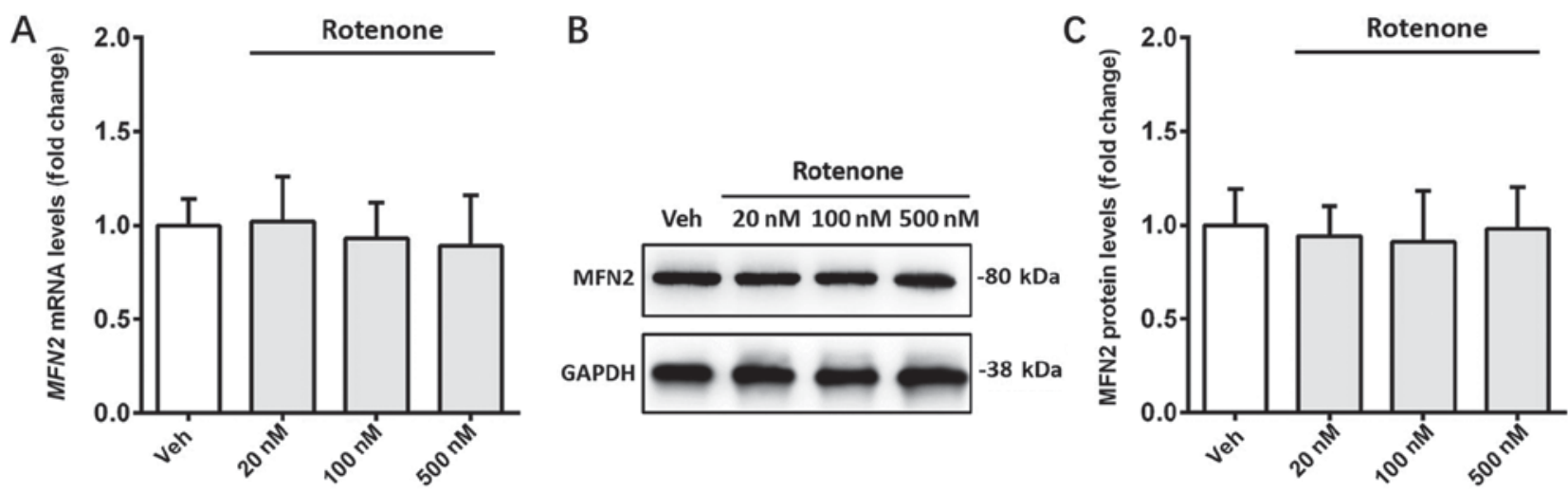

Figure 2. Expression of MFN2 in SH-SY5Y cells is stable following treatment with rotenone. SH-SY5Y cells were incubated with different doses of rotenone $(20,100$ and $500 \mathrm{nM})$ for $12 \mathrm{~h}$. (A) mRNA expression levels of MFN2 were detected by reverse transcription-quantitative polymerase chain reaction. Data were expressed as a fold change relative to vehicle control group. GAPDH was used as an internal control. Protein expression levels of MFN2 were (B) detected by western blotting and (C) quantified. Data are presented as a fold change relative to the vehicle control group. GAPDH was used as a loading control. All data were analyzed by one-way analysis of variance followed by Tukey's post hoc test. Values are presented as the mean \pm standard deviation. MFN2, mitofusin-2; veh, vehicle control.

treatment with rotenone, compared with the control shRNA group (Fig. 3D). Knockdown of MFN2 did not significantly affect the viability of SH-SY5Y cells untreated with rotenone (data not shown). Taken together, the above results indicated that MFN2 knockdown exacerbated cell apoptosis caused by rotenone, suggesting that MFN2 may serve a protective role against rotenone-induced cell apoptosis.

Overexpression of MFN2 ameliorates apoptosis induced by rotenone in $\mathrm{SH}-\mathrm{SY} 5 \mathrm{Y}$ cells. To validate the protective role of MFN2 against rotenone-induced cell apoptosis, MFN2 was overexpressed in SH-SY5Y cells using a lentiviral strategy. The alterations in MFN2 protein levels were confirmed by western blotting (Fig. 4A and B). Subsequently, cells were incubated with $100 \mathrm{nM}$ rotenone for $12 \mathrm{~h}$. The increase in caspase-3 activity induced by rotenone was attenuated by MFN2 overexpression ( $\mathrm{P}<0.05$; Fig. 4C). Furthermore, overexpression of MFN2 ameliorated the rotenone-induced reduction in cell viability $(\mathrm{P}<0.05$; Fig. 4D). MFN2 overexpression did not significantly affect the viability of SH-SY5Y cells without rotenone treatment (data not shown).

\section{Discussion}

Rotenone, a mitochondrial complex I inhibitor, was reported to reproduce a number of neuropathological features of PD, including loss of dopaminergic neurons (19). In the present study, rotenone resulted in decreased viability of human neuroblastoma SH-SY5Y cells, in a dose-dependent manner. Furthermore, expression levels of caspase-3, a crucial executioner of apoptosis, were markedly increased following treatment with rotenone, suggesting that apoptosis contributed to the loss of SH-SY5Y cells. These results are in agreement with previous reports by the authors of the present study, where rotenone induced apoptosis in human and mouse dopaminergic cell lines $(13,15)$. It has been previously demonstrated that apoptosis 
A

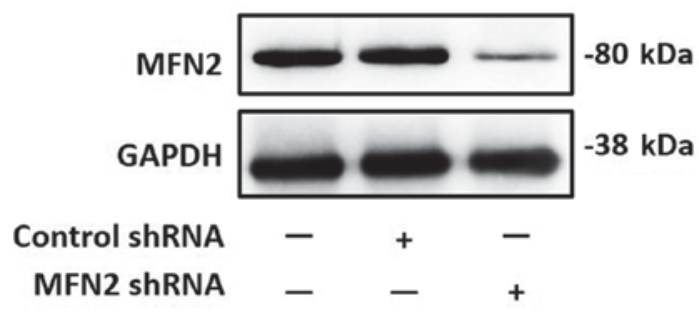

C



B

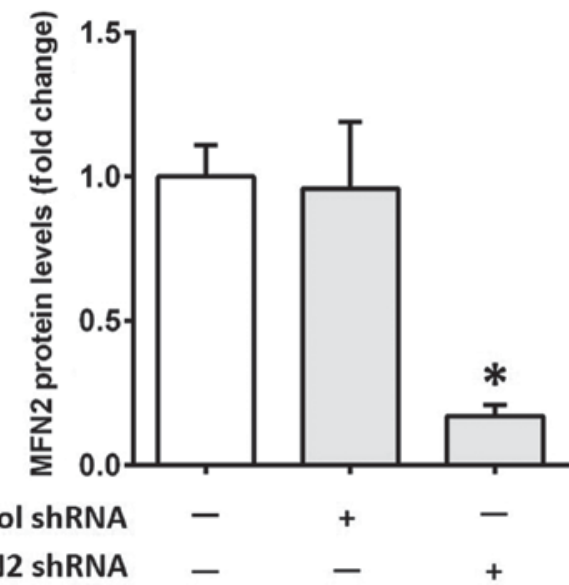

D

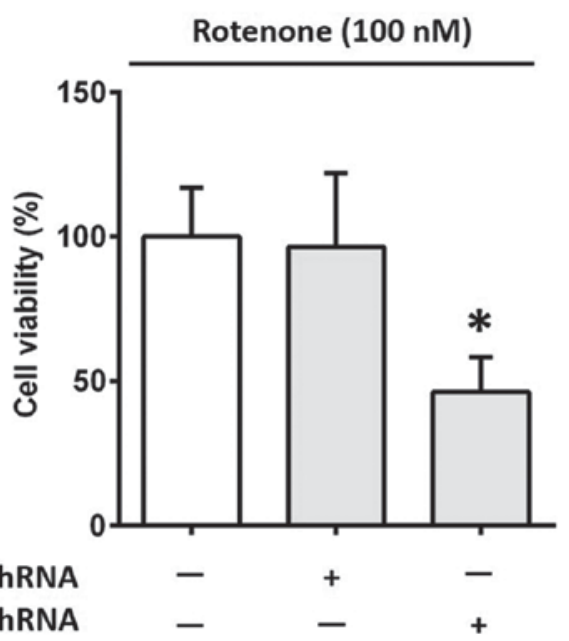

Figure 3. Knockdown of MFN2 exacerbates apoptosis induced by rotenone in SH-SY5Y cells. A lentiviral strategy was used to knock down the expression of MFN2 in SH-SY5Y cells. (A) The alterations in MFN2 protein levels were confirmed by western blotting. (B) The protein expression levels were quantified. Subsequently, the SH-SY5Y cells were incubated with $100 \mathrm{nM}$ rotenone for $12 \mathrm{~h}$. (C) A colorimetric assay was used to detect the activity of caspase-3 in SH-SY5Y cells. Data are presented as a fold change relative to untransfected cells. (D) MTT assay was used to evaluate the viability of SH-SY5Y cells. Data are presented as a percentage of the untransfected group. All data were analyzed by one-way analysis of variance followed by Tukey's post hoc test. Values are presented as the mean \pm standard deviation. " $\mathrm{P}<0.05$ vs. the control shRNA group. shRNA, short hairpin RNA; MFN2, mitofusin-2.

is closely modulated by two pathways: The receptor-dependent apoptotic pathway and the mitochondria-mediated apoptotic pathway (5). Recently, the authors of the present study suggested that apoptosis of dopaminergic neurons was primarily achieved via the mitochondria-mediated apoptotic pathway, since cytochrome c, a mitochondrial pro-apoptotic factor, was observed in the cytoplasm of dopaminergic neurons (6). However, the underlying molecular mechanisms remain unclear.

MFN2, a mitochondrial protein that belongs to the family of GTPases, is widely distributed in numerous tissues and organs including heart, kidney, liver, and brain (7). At the subcellular level, MFN2 is located predominantly in the outer membrane of the mitochondria (8). A previous study has indicated that MFN2 was required for several physiological processes of mitochondria including fusion and metabolism (9). Furthermore, several lines of evidence indicated that MFN2 may be involved in the process of mitochondria-mediated apoptosis and participated in the pathogenesis of several neurological disorders (10-12). Loss-of-function mutations of MFN2 gene led to the onset of CMT type 2A, a neurological disease characterized by the degeneration of axons in the peripheral nervous system (20). In an in vitro model of ischemic stroke, decreased MFN2 expression was closely associated with increased neuronal apoptosis (21). In addition, reduced MFN2 level was associated with increased oxidative stress and apoptosis of neurons during neurodegeneration $(22,23)$. In the present study, knockdown of MFN2 in a cellular model of $\mathrm{PD}$ induced by rotenone aggravated cell apoptosis. The above results implied a protective role of MFN2 against apoptosis. To further validate the antiapoptotic effect of MFN2 in the in vitro model of $\mathrm{PD}, \mathrm{MFN} 2$ was overexpressed in SH-SY5Y cells prior to treatment with rotenone. For the first time to the best of the authors' knowledge, it was demonstrated that MFN2 ameliorated apoptosis induced by rotenone. This observation was supported by previous reports that MFN2 overexpression attenuated neuronal apoptosis under the conditions of ischemic stroke or neurodegeneration (21-23), indicating the antiapoptotic effect of MFN2.

The present study has certain limitations. The antiapoptotic effect of MFN2 was evaluated in a cellular model of PD. Therefore, the results require further confirmation in vivo using animal models of PD. Additionally, the precise signaling 
A

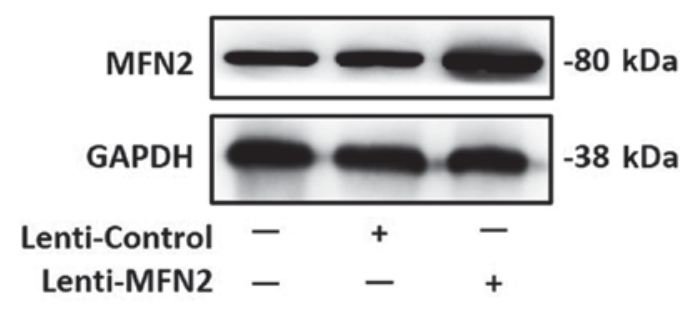

C

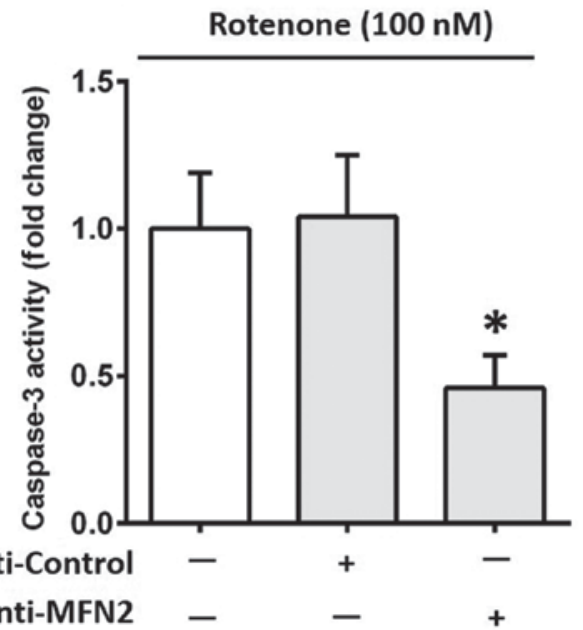

B

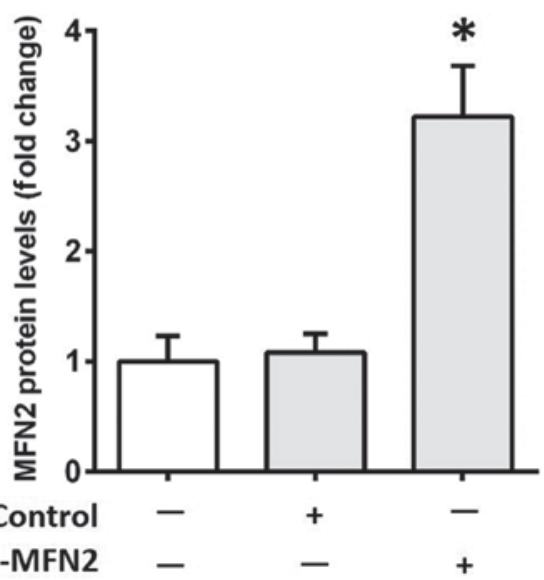

D

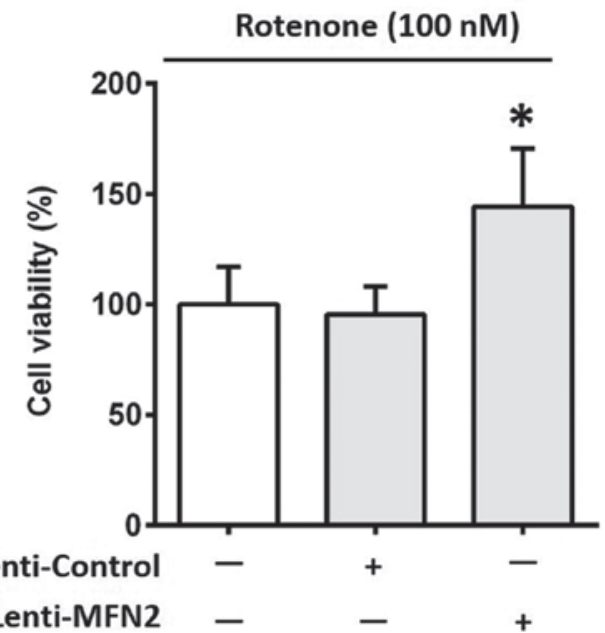

Figure 4. Overexpression of MFN2 ameliorates apoptosis induced by rotenone in SH-SY5Y cells. A lentiviral strategy was used to overexpress MFN2 in SH-SY5Y cells. The alterations in MFN2 protein levels were (A) confirmed by western blotting and (B) quantified. Subsequently, the SH-SY5Y cells were incubated with $100 \mathrm{nM}$ rotenone for $12 \mathrm{~h}$. (C) A colorimetric assay was used to detect the activity of caspase-3 in SH-SY5Y cells. Data are presented as a fold change relative to untransfected cells. (D) MTT assay was used to evaluate the viability of SH-SY5Y cells. Data are presented as a percentage of the untransfected group. All data were analyzed by one-way analysis of variance followed by Tukey's post hoc test. Values are presented as the mean \pm standard deviation. ${ }^{*} \mathrm{P}<0.05$ vs. the control lentivirus group. Lenti, lentivirus; MFN2, mitofusin-2.

underlying the antiapoptotic effect of MFN2 was not identified in the present study and this should be investigated in the future.

In conclusion, the present study indicated that the expression of MFN2 was stable following treatment with rotenone in a cellular model of PD. Using a lentiviral knockdown and overexpression strategy, it was demonstrated that MFN2 prevented rotenone-induced cell death by amelioration of apoptosis. These results revealed a protective role of MFN2 against apoptosis in an in vitro model of PD and may be used to establish MFN2 as a potential therapeutic target for the treatment of this disease.

\section{Acknowledgements}

Not applicable.

\section{Funding}

The present study was supported by National Natural Science Foundation of China (grant no. 81771140), Natural Science Foundation of Jiangsu Province (grant no. BK20151084),
Key Research and Development Project of Jiangsu Province (grant no. BL2014014), 'Six Talent Summit' Foundation of Jiangsu Province (grant no. 2016-WSN-180), Youth Medical Talent Program of Jiangsu Province (grant no. QNRC2016068), Medical Innovation Team of Jiangsu Province (grant no. CXTDA2017030), and Nanjing Medical Science and Technology Development Foundation for Distinguished Young Scholars (grant no. JQX17008).

\section{Availability of data and materials}

The datasets used and/or analyzed during the present study are available from the corresponding author on reasonable request.

\section{Authors' contributions}

YZ and TJ designed the present study. YY and LX performed the experiments. $\mathrm{ZO}$ and $\mathrm{XX}$ analyzed the data and prepared all figures. TJ wrote the manuscript. All authors have read and approved this manuscript. 


\section{Ethics approval and consent to participate}

Not applicable.

\section{Patient consent for publication}

Not applicable.

\section{Competing interests}

The authors declare that they have no competing interests.

\section{References}

1. Tysnes OB and Storstein A: Epidemiology of Parkinson's disease. J Neural Transm (Vienna) 124: 901-905, 2017.

2. Schapira AH: Neurobiology and treatment of Parkinson's disease. Trends Pharmacol Sci 30: 41-47, 2009.

3. Gao Q, Jiang T, Zhao HR, Wu L, Tian YY, Ou Z, Zhang L, Pan Y, $\mathrm{Lu} \mathrm{J}$ and Zhang YD: Activation of autophagy contributes to the Angiotensin II-triggered apoptosis in a dopaminergic neuronal cell line. Mol Neurobiol 53: 2911-2919, 2016.

4. Zhao HR, Jiang T, Tian YY, Gao Q, Li Z, Pan Y, Wu L, Lu J and Zhang YD: Angiotensin II triggers apoptosis via enhancement of NADPH Oxidase-dependent oxidative stress in a dopaminergic neuronal cell line. Neurochem Res 40: 854-863, 2015.

5. Green DR and Llambi F: Cell death signaling. Cold Spring Harb Perspect Biol 7: pii: a006080, 2015.

6. Ou Z, Jiang T, Gao Q, Tian YY, Zhou JS, Wu L, Shi JQ and Zhang YD: Mitochondrial-dependent mechanisms are involved in angiotensin II-induced apoptosis in dopaminergic neurons. J Renin Angiotensin Aldosterone Syst 17: pii, 2016.

7. Schrepfer E and Scorrano L: Mitofusins, from mitochondria to metabolism. Mol Cell 61: 683-694, 2016.

8. de Brito OM and Scorrano L: Mitofusin 2: A mitochondriashaping protein with signaling roles beyond fusion. Antioxid Redox Signal 10: 621-633, 2008.

9. Zorzano A, Hernández-Alvarez MI, Sebastián D and Muñoz JP: Mitofusin 2 as a driver that controls energy metabolism and insulin signaling. Antioxid Redox Signal 22: 1020-1031, 2015.

10. Stuppia G, Rizzo F, Riboldi G, Del Bo R, Nizzardo M, Simone C, Comi GP, Bresolin N and Corti S: MFN2-related neuropathies: Clinical features, molecular pathogenesis and therapeutic perspectives. J Neurol Sci 356: 7-18, 2015.

11. Shi Y, Yi C, Li X, Wang J, Zhou F and Chen X: Overexpression of Mitofusin2 decreased the reactive astrocytes proliferation in vitro induced by oxygen-glucose deprivation/reoxygenation. Neurosc Lett 639: 68-73, 2017.
12. Wang X, Su B, Lee HG, Li X, Perry G, Smith MA and Zhu X: Impaired balance of mitochondrial fission and fusion in Alzheimer's disease. J Neurosci 29: 9090-9103, 2009.

13. Wu L, Luo N, Zhao HR, Gao Q, Lu J, Pan Y, Shi JP, Tian YY and Zhang YD: Salubrinal protects against rotenone-induced SH-SY5Y cell death via ATF4-parkin pathway. Brain Res 1549: 52-62, 2014.

14. Jiang T, Tan L, Zhu XC, Zhang QQ, Cao L, Tan MS, Gu LZ, Wang HF, Ding ZZ, Zhang YD and Yu JT: Upregulation of TREM2 ameliorates neuropathology and rescues spatial cognitive impairment in a transgenic mouse model of Alzheimer's disease. Neuropsychopharmacology 39: 2949-2962, 2014.

15. Lu J, Wu L, Jiang T, Wang Y, Zhao H, Gao Q, Pan Y, Tian Y and Zhang Y: Angiotensin AT2 receptor stimulation inhibits activation of NADPH oxidase and ameliorates oxidative stress in rotenone model of Parkinson's disease in CATH.a cells. Neurotoxicol Teratol 47: 16-24, 2015.

16. Jiang T, Zhang YD, Chen Q, Gao Q, Zhu XC, Zhou JS, Shi JQ, $\mathrm{Lu} \mathrm{H}$, Tan L and Yu JT: TREM2 modifies microglial phenotype and provides neuroprotection in P301S tau transgenic mice. Neuropharmacology 105: 196-206, 2016.

17. Jiang T, Zhang YD, Gao Q, Zhou JS, Zhu XC, Lu H, Shi JQ, Tan L, Chen Q and Yu JT: TREM1 facilitates microglial phagocytosis of amyloid beta. Acta Neuropathol 132: 667-683, 2016.

18. Livak KJ and Schmittgen TD: Analysis of relative gene expression data using real-time quantitative PCR and the 2(-Delta Delta C(T)) method. Methods 25: 402-408, 2001.

19. Johnson ME and Bobrovskaya L: An update on the rotenone models of Parkinson's disease: Their ability to reproduce the features of clinical disease and model gene-environment interactions. Neurotoxicology 46: 101-116, 2015.

20. Bombelli F, Stojkovic T, Dubourg O, Echaniz-Laguna A, Tardieu S, Larcher K, Amati-Bonneau P, Latour P, Vignal O, Cazeneuve C, et al: Charcot-Marie-Tooth disease type 2A: From typical to rare phenotypic and genotypic features. JAMA Neurol 71: 1036-1042, 2014.

21. Peng C, Rao W, Zhang L, Wang K, Hui H, Wang L, Su N, Luo P, Hao YL, Tu Y, et al: Mitofusin 2 ameliorates hypoxia-induced apoptosis via mitochondrial function and signaling pathways. Int J Biochem Cell Biol 69: 29-40, 2015.

22. Park J, Choi H, Min JS, Kim B, Lee SR, Yun JW, Choi MS, Chang KT and Lee DS: Loss of mitofusin 2 links beta-amyloidmediated mitochondrial fragmentation and Cdk5-induced oxidative stress in neuron cells. J Neurochem 132: 687-702, 2015.

23. Zhao F, Wang W, Wang C, Siedlak SL, Fujioka H, Tang B and Zhu X: Mfn2 protects dopaminergic neurons exposed to paraquat both in vitro and in vivo: Implications for idiopathic Parkinson's disease. Biochim Biophy Acta 1863: 1359-1370, 2017. 